ARTíCULO De REVISIÓN

\title{
Aportes desde las neurociencias para la comprensión de los procesos de aprendizaje en los contextos educativos
}

\section{Contributions from the Neurosciences for the Understanding of Learning Processes in Educational Contexts}

\author{
Sebastián C. Araya-Pizarro \\ Universidad de La Serena, La Serena, Chile \\ ORCID: https://orcid.org/0000-0002-5857-8441 \\ Laura Espinoza Pastén* \\ Universidad de La Serena, La Serena, Chile \\ ORCID: https://orcid.org/0000-0002-0947-0039
}

Recibido 16-04-19 Revisado 30-05-19 Aprobado 04-11-19 En línea 07-11-19

*Correspondencia

Email: laura.espinozap@userena.cl
Citar como:

Araya-Pizarro, S.C., \& Espinoza Pastén, L. (2020) Aportes desde las neurociencias para la comprensión de los procesos de aprendizaje en los contextos educativos. Propósitos y Representaciones, 8(1), e312. doi: http://dx.doi.org/10.20511/pyr2020.v8n1.312

(C) Universidad San Ignacio de Loyola, Vicerrectorado de Investigación, 2020 


\section{Resumen}

En la actual literatura científica se alude a los aportes desde las Neurociencias para la comprensión de los procesos de aprendizaje. Es así, como también desde las ramas de las Neurociencias tales como la Neurociencia Cognitiva, nacen aportes específicos que facilitan la comprensión de estos procesos. Por otra parte, la Neuroeducación como nueva disciplina propone tomar estos aportes desde las Neurociencias para la mejora de las prácticas educativas, y con ello optimizar los aprendizajes. El presente artículo tiene como objetivo analizar los aportes teóricos vigentes desde las Neurociencias, para comprender el aprendizaje situado en los contextos educativos. Para ello, se alude a la relación de los conceptos Neurociencias, Neurociencia Cognitiva y Neuroeducación. Además, se analizan los principales aportes desde las Neurociencias para comprender los procesos de neuroplasticidad, y el impacto de las emociones, la calidad del sueño, la actividad física y los contextos sociales en el aprendizaje. Por último, se discute sobre la importancia de estos aportes y de qué modo orientan el rol docente y las prácticas educativas para que permitan el alcance de aprendizajes significativos.

Palabras clave: Aprendizaje; Cognición; Contexto de aprendizaje; Investigación sobre el cerebro.

\section{Summary}

In the current scientific literature refers to the contributions from the Neurosciences for the understanding of learning processes. This is how, as well as from the branches of Neurosciences, such as Cognitive Neuroscience, specific contributions are born that facilitates the understanding of these processes. On the other hand, Neuroeducation as a new discipline proposes to take these contributions from the Neurosciences for the improvement of educational practices, and thereby optimize learning. The objective of this article is to analyze the theoretical contributions in force from the Neurosciences, to understand the learning situated in educational contexts. For this, the relationship of the concepts Neuroscience, Cognitive Neuroscience and Neuroeducation is alluded to. In addition, the main contributions from the Neurosciences are analyzed to understand the processes of neuroplasticity, and the impact of emotions, sleep quality, physical activity and social contexts in learning. Finally, we discuss the importance of these contributions and how they guide the teaching role and educational practices so that they allow for meaningful learning.

Keywords: Learning, Cognition, Learning Context, Brain Research.

\section{Introducción}

El proceso de aprendizaje ha sido estudiado a lo largo de la historia desde diferentes disciplinas, tales como la filosofía, la psicología y otras afines. Asimismo, es el elemento central que moviliza los procesos educativos. En la actualidad, el avance científico y la tecnología han permitido que los estudios sobre el aprendizaje sean abundantes, sobre todo vinculados al sustrato de dicho complejo proceso: el cerebro. Las investigaciones efectuadas desde las variadas ramas de las Neurociencias han generado contribuciones abundantes, aunque específicas, que en ocasiones no se articulan directamente con los procesos educativos. Sin embargo, de manera reciente se ha evidenciado el esfuerzo desde diversos autores, quienes buscan vincular los aportes desde estas ciencias con el contexto educativo. Esta vinculación permite tomar dichos hallazgos para alcanzar la comprensión de los procesos de aprendizaje, así como tomar consideraciones que permitan mejorar dichos procesos. 
El siguiente artículo consiste en un análisis teórico que considera los diferentes aportes que la evidencia científica da desde las Neurociencias, y sobre todo desde la Neurociencia Cognitiva y la Neuroeducación para comprender los procesos de aprendizaje, vinculados al ámbito de la Educación. La composición de este artículo se llevó a cabo desde una revisión documental específica centrada en este tema, considerando publicaciones de revistas indexadas y libros actuales afines.

La necesidad de contar con una revisión bibliográfica que vincule los aportes entre Neurociencias y Educación permite establecer un puente entre ambas áreas. Este puente es el Aprendizaje. Dicho proceso es la meta para lograr en los contextos educativos, mientras que para las Neurociencias -y sobre todo para la Neurociencia Cognitiva y la Neuroeducación- es el objeto de estudio. Algunos autores han hecho esfuerzos para establecer este vínculo, considerando las limitaciones propias de cada área a nivel de naturaleza y lenguaje (Goswami, 2015). Sin embargo, estas publicaciones aún son escasas, sobre todo en idioma español. A partir de los aportes de este tipo de revisiones se puede masificar la difusión de las relaciones entre estas áreas, que usualmente se visualizan como distantes, pero que en realidad deben complementarse para favorecer los procesos de enseñanza y aprendizaje del estudiantado. Asimismo, para la difusión de orientaciones que permitan generar herramientas para optimizar los aprendizajes, en base a la evidencia desde las Neurociencias.

En este artículo, primeramente, se considera la conceptualización y relación entre los términos Neurociencias, Neurociencia Cognitiva y Educación y su vinculación con el aprendizaje en contextos educativos. En segundo lugar, se analizan los principales aportes desde las Neurociencias para comprender los procesos de neuroplasticidad y cómo los entornos de aprendizaje influyen en ello. Posteriormente, se alude a los aportes para comprender la relevancia de las emociones tanto positivas como negativas en la calidad de los aprendizajes. En cuarto lugar, se analiza cómo una adecuada higiene del sueño y la actividad física contribuyen al alcance de aprendizajes de mayor calidad. Luego, se plantean los aportes para comprender el rol de las neuronas espejo para el aprendizaje en contextos sociales. Por último, se efectúa una discusión y las conclusiones, referidas a la importancia de estas contribuciones neurocientíficas para la comprensión y la optimización de los aprendizajes, considerando tanto el rol docente y las prácticas de aula como elementos clave.

\section{Neurociencias, Neurociencia Cognitiva y Neuroeducación ¿Cómo se vinculan estos conceptos?}

Las Neurociencias son el conjunto de ciencias y disciplinas científicas y académicas que estudian el sistema nervioso, centrando su atención en la actividad del cerebro y su relación e impacto en el comportamiento (Gago \& Elgier, 2018). Se presenta como una rama de investigación bastante reciente cuyo origen se remonta a la década de 1960 (Borck, 2016), abordando aspectos neurobiológicos de la conducta apoyados en la psicología cognitiva, la lingüística, la antropología y la inteligencia artificial, entre otros. Además, se trata de un conjunto de ciencias cuyo interés por ser estudiadas ha aumentado durante la última década del siglo XX (Martín-Loeches, 2015). Desde las Neurociencias se identifican varias ciencias y disciplinas vinculadas, tales como la neurobiología, neurofisiología, neuropsicología, neuroquímica, neuroanatomía, neuromarketing, neuroliderazgo, neuroeconomía, neuromanagement, neurogenética, neurociencia computacional, entre otras (Barrios, 2016; Braidot, 2013; Leopoldo \& Joselevitch, 2018). Asimismo, se ha iniciado su aplicación en diferentes campos como la filosofía, la ética, la sociología, el arte, la economía e incluso la educación (Comins-Mingol \& París-Albert, 2013).

En tal contexto, destacan dos conocidas líneas de trabajo vinculadas a las Neurociencias y el Aprendizaje: la Neurociencia Cognitiva y la Neuroeducación. La primera, puede 
comprenderse como la rama de las Neurociencias dedicada a estudiar las relaciones entre el sistema nervioso y la cognición humana. Por ello suelen describirla también como un área científica que nace de la convergencia de dos disciplinas: la Psicología Cognitiva que estudia las funciones mentales superiores y las Neurociencias, que estudian el sistema nervioso que las sustenta (Redolar, 2013). Por otra parte, y según la literatura reciente, la Neuroeducación se entiende como aquella disciplina que se ocupa de indagar y difundir sobre la optimización del proceso de enseñanza y aprendizaje con base en el funcionamiento del cerebro y los fundamentos neurobiológicos que lo sustentan. Por lo tanto, su propósito esencial sería el de aplicar sus hallazgos al mejoramiento del proceso educativo, buscando comprender cómo el cerebro cambia y se adapta durante el aprendizaje (Mora, 2017; Valerio, Jaramillo, Caraza \& Rodríguez, 2016).

Lo expuesto pone de manifiesto la fuerte relación existente entre las dos ramas vinculadas a las Neurociencias. Ambas se enfocan en indagar y comprender cómo aprende el cerebro. Por tanto, pueden contribuir, en gran manera, a proporcionar nuevas técnicas para potenciar los procesos de aprendizaje y del desarrollo cognitivo, sus mecanismos causales, las variables que los afectan y una manera práctica de analizar la eficacia de diferentes pedagogías que conlleven a una formación equitativa y de calidad (Goswami, 2015). También, resultaría clave para ayudar a resolver los problemas neurofuncionales que afectan a los estudiantes en todas las etapas del sistema educativo (Blanco, Miguel, García-Castellón \& Martín, 2017).

Para que el proceso de enseñanza-aprendizaje sea efectivo se requiere intencionar el aprendizaje de los aprendices, considerando los componentes cognitivos, así como los emocionales; por ejemplo: el interés respecto de lo que están aprendiendo. En tal escenario, la Neurociencia Cognitiva y la Neuroeducación se instauran según la literatura actual como áreas potenciales para optimizar el diseño y estrategias educativas al brindar lineamientos para el mejoramiento de la enseñanza-aprendizaje, en base al estudio tanto teórico como empírico de los procesos mentales del cerebro, tales como el pensamiento, la memoria, la atención y procesos de percepción complejos.

Valerio et al. (2016) sintetizan una serie de prácticas que diversos investigadores de las Neurociencias proponen para poder ser utilizadas dentro del salón de clases. Por ejemplo, en relación con la atención, utilizar pausas en los niveles de atención que den tiempo de asimilar cada nuevo aprendizaje. En cuanto a la motivación, desarrollar actividades placenteras que reduzcan el estrés y propicien la curiosidad y perseverancia. Para la memoria, recomiendan las repeticiones en distintos escenarios que facilitan la memorización duradera y activar un conocimiento ya almacenado que permita conectar el nuevo conocimiento, entre otras.

\section{La base para el aprendizaje constante: La plasticidad neural}

La plasticidad neural consiste en la capacidad de las diferentes redes neuronales de nuestro cuerpo para modificarse a lo largo de nuestro desarrollo ontogenético (Gago \& Elgier, 2018). Por tanto, la neuroplasticidad reconoce el papel fundamental que el ambiente ejerce en la modulación de la actividad genética, permitiéndole al sujeto realizar una reconstrucción propia a partir de la interacción del genoma con el ambiente. Es importante comprender, que dicha flexibilidad de las neuronas es de conexión, desconexión y reconexión constante, dependiendo fundamentalmente de cuán consolidadas se encuentren estas redes interconectadas y cuánto se usen en la vida cotidiana.

Más específicamente, la neuroplasticidad puede entenderse como la potencialidad del sistema nervioso de modificarse tanto anatómica como fisiológicamente para formar conexiones nerviosas en respuesta a la información nueva, la estimulación sensorial, el desarrollo, la disfunción o el daño (Garcés-Viera \& Suarez-Escudero, 2014). Es decir, la plasticidad cerebral 
se origina por y para responder a procesos adaptativos que son frutos de la estimulación ambiental (Justel \& Diaz, 2012).

Considerando los antecedentes expuestos, es posible comprender que educar es cambiar las posibilidades del cerebro. Por tanto, magnifica la relevancia de la labor docente en el desarrollo efectivo del proceso de formación del estudiantado. Ello evidencia que el éxito formativo no nace espontáneamente por la masificación de un tipo de enseñanza única y de carácter reduccionista, sino que el sistema educativo debería brindar la posibilidad de ajuste a las diversas maneras de aprendizaje de las personas bajo un modelo educativo centrado en el bienestar de sus participantes.

No obstante, es importante destacar que aún cuando la educación y el aprendizaje cambian y afectan de manera importante las bases biológicas del cerebro, no se pueden desatender otros elementos que intervienen sobre el aprendizaje, el desarrollo cerebral y la propia formación del ser humano. Tanto el ámbito educativo en todos sus niveles, así como el ambiente sociocultural cotidiano en el cual se desarrolla la mayor parte de los actores del proceso educativo, deben contribuir conjuntamente al desarrollo integral que persigue la educación (Barrios, 2016). Así, no solo se debería tener conciencia de la relación entre educación y aprendizaje con las bases neuronales y biológicas de estos procesos. También se debería conocer y considerar estrategias para fortalecer aspectos del entorno sociocultural que influyen en el desarrollo cerebral de los educandos, los cuales podrían ser causantes de desequilibrios y/o brechas educacionales.

\section{El aprendizaje con base en las emociones}

Las Neurociencias han demostrado que las emociones positivas facilitan la memoria y el aprendizaje pues ayudan a mantener la curiosidad y la motivación, condiciones trascendentales para un aprendizaje efectivo y duradero (Mora, 2017). A nivel neurofisiológico, las emociones activan el hipocampo -que está relacionado con la memoria y el aprendizaje-, anclando mejor los conocimientos obtenidos. De este modo, produce recuerdos de tipo emocional con la mediación de la amígdala cerebral, facilitando su posterior evocación. Existe, así, un entrecruzamiento entre la emoción y la memoria que genera recuerdos fuertemente sostenidos en la memoria a largo plazo, con mayores posibilidades de ser recuperado y por tanto consolidado.

Si las emociones que se asocian a la experiencia de aprendizaje son de carácter negativo -tales como la ansiedad, el miedo, el nerviosismo, la preocupación, la tristeza, la ira- actuarían como barreras del proceso enseñanza-aprendizaje. Específicamente, entorpecerían el anclaje de los conocimientos nuevos en la mente debido a la liberación de la hormona del estrés o cortisol. De forma crónica, estas situaciones negativas podrían causar trastornos en los procesos cognitivos fundamentales para el rendimiento académico y el aprendizaje de competencias para la posterior vida laboral.

La emocionalidad influye significativamente en el aprendizaje de una persona, pues ellas condicionan las acciones posibles para su alcance (Pacheco, Villagran \& Guzmán, 2015). Asimismo, las emociones son las que determinan la toma de decisiones en la zona prefrontal del cerebro (Meltzer, 2018). Por tanto, si las emociones son no gratas, afectarían negativamente la predisposición a aprender cosas nuevas o a establecer contactos con otros, impidiendo al estudiante generar conexiones con los demás.

A modo de reflexión, es importante considerar la enseñanza misma como una experiencia emocional en la que intervienen tanto procesos cognitivos como afectivos. La práctica de la enseñanza implica una gran cantidad de trabajo emocional que compromete 
nuestro sentir en las interacciones relevantes que se dan con nuestros estudiantes, familiares y compañeros (Dávila, Borrachero, Brígido \& Costillo, 2014).

Por ello, y respecto al rol docente, es importante desarrollar la capacidad de actuar para transformar y autorregular las emociones en el propio aprendizaje, tanto en los aprendices como en los enseñantes (Brígido, Caballero, Bermejo, Luisa, \& Mellado, 2009). Todo profesor actúa como un factor motivacional dual, que puede aumentar, promover, restringir o desmotivar al estudiante. Dichas acciones pueden causar incluso frustración, insatisfacción o resentimiento (González, 2006).

\section{Actividad física y calidad del sueño para fortalecer el aprendizaje}

La actividad física es un tema de sumo interés para la Neuroeducación, que puede traer aparejada una mejora general de las funciones cognitivas, mayor autoestima, y beneficiar a personas diagnosticadas con Trastorno por Déficit de Atención e Hiperactividad (TDAH), ansiedad o depresión, además de prevenir el síndrome de Burnout en estudiantes (Gago \& Elgier, 2018). El ejercicio también mejora la predisposición física y psicológica hacia el aprendizaje, aumentando los niveles de motivación y atención (Blakemore \& Frith, 2007). Las autoras plantean que a nivel neuronal el ejercicio físico actúa como un neuroprotector de ciertos tipos de células cerebrales y que el estrés celular leve que produce el ejercicio genera mayores grados de motivación y activación a lo largo de las clases, mejorando el nivel académico y disminuyendo los niveles de estrés.

Existe vasta evidencia científica que confirma cómo favorece la actividad física y mejora los procesos cognitivos durante la niñez y la adolescencia (Sibley \& Etnier, 2003). Algunos de estos estudios explican tales beneficios argumentando que la práctica de actividad física genera un aumento del flujo sanguíneo cerebral, cambios en la liberación de neurotransmisores, cambios estructurales en el sistema nervioso central y altos niveles de excitación (Hernández \& Portolés, 2016).

Respecto de la calidad del sueño, dormir adecuadamente también reduce los niveles de estrés y promueve la capacidad memorística, siendo un factor preponderante para el aprendizaje. En efecto, en la actualidad diversas investigaciones, tanto experimentales como clínicas, han demostrado que el sueño tiene efectos positivos sobre distintos tipos de memoria (Carillo-Mora, Ramírez-Peris, \& Magaña-Vásquez, 2013). Según la literatura, esto se debería al ritmo circadiano de cada persona, el cual se encuentra asociado a los cambios de luz-oscuridad que se dan durante el día y la noche. Es en esta fase de sueño cuando las neuronas generan procesos de limpieza de las toxinas, regeneración celular y consolidación de los aprendizajes (Blackemore y Frith, 2007). Cuando los hábitos del sueño se ven desregulados pueden surgir trastornos del sueño, perjudicando así el rendimiento de la persona al afectar distintas funciones cognitivas. Algunas disfunciones posibles son: alteración de la capacidad de juicio, disminución de la flexibilidad cognitiva, alteraciones del humor -irritabilidad y enojo-, fatiga excesiva y presencia de "microsueños" involuntarios, el tiempo de reacción se prolonga, aparece el enlentecimiento cognitivo, entre otros (Carillo-Mora et al., 2013).

En el ámbito estudiantil estos problemas son particularmente comunes en los adolescentes y jóvenes universitarios, segmentos etarios más proclives a presentar una baja calidad de sueño dado que suelen dormirse muy tarde. Dicha situación genera conflicto con sus labores académicas matutinas, especialmente cuando las materias más complejas de estudiar se ubican al principio de la jornada de clases. Estos aspectos nos hacen reflexionar sobre la importancia del sueño para el aprendizaje y la necesidad de establecer acciones para la prevención de las alteraciones del sueño -medidas de higiene del sueño-, direccionados a mejorar la calidad de vida de nuestros estudiantes. 


\section{Neuronas espejo y aprendizaje en contextos sociales}

Las neuronas espejo -también conocidas como neuronas especulares o neuronas cubelli-, son un tipo de neuronas que nos permiten comprender a los demás y nos vinculan con la postura emocional de la otra persona. Son neuronas que se activan al realizar cierta acción y al observar las acciones de un semejante, entregando un entendimiento neurobiológico de la empatía y la teoría de la mente (Arboccó de los Heros, 2016; Gago \& Elgier, 2018). Por ende, se supondría que son las precursoras del aprendizaje por imitación, la interacción del yo y la comprensión social, las actitudes, el prejuicio, el consenso social, el rechazo social y la toma de decisiones sociales (Pineda, 2016). Todas estas son procesos claves que caracterizan las relaciones humanas e identifican a los seres humano como seres sociales capaces de desarrollar un comportamiento empático y comprensivo de las emociones de los demás. Esto facilita el aprendizaje cooperativo, la adquisición de pautas de comportamiento y la transmisión de la cultura. Se cree que sin la existencia de las neuronas espejo, la imitación sería simplemente una reproducción mecánica de lo observado. Asimismo, que no atendería al estado emocional y/o mental de los demás, dificultando la capacidad de ser conscientes sobre lo que están experimentando otras personas. Además, que afectaría la comunicación efectiva. En las organizaciones educacionales la comunicación es un proceso clave para la gestión educativa. Implica el cumplimiento de reglas, normas, políticas y lineamientos indispensables para la obtención de un ambiente de armonía y estabilidad, facilitando el trabajo en grupo (Romero, Monzan \& Hernández, 2014).

Por ende, comprender cómo funcionan las neuronas espejo no solo es útil para mejorar el rol de los agentes educativos y la convivencia escolar. También ayudaría a deliberar estrategias potenciales para solucionar problemas que pueden causar disfunciones en el plano relacional -profesor-aprendiz, aprendiz-aprendiz-. El aprendizaje, como se ha revisado a lo largo de este artículo, no solamente depende de la condición genética propia de cada individuo, sino que también del entorno próximo y del contexto socio-cultural en el que se desarrolla. De esta forma, los profesores pueden idear estrategias para generar en el aula climas emocionales positivos que fomenten el aprendizaje compartido, en que unos y otros colaboran cooperativamente en pos del progreso general del curso. También, considerando el rol de docentes como referentes, se posibilitaría guiar el aprendizaje de los estudiantes y establecer pautas o modelos para la resolución de problemas. Esta guía o mediación no debe incluir solamente la enseñanza de contenidos temáticos teóricos, sino que también la integración de ideas, valores y actitudes, tan importantes como los conocimientos impartidos.

\section{Discusión y conclusiones sobre los aportes desde las Neurociencias para la comprensión de los procesos de aprendizaje}

Los aportes desde las Neurociencias son de suma importancia para comprender y buscar mejorar los procesos de enseñanza-aprendizaje. Actualmente se reconoce que el rol docente es esencial dentro del proceso formativo de los estudiantes pues no se limita a una mera transferencia de conocimientos, sino que el proceso interactivo alumno-profesor produce cambios a nivel biológico, cognitivo y emocional. Y precisamente, bajo las condiciones y contexto del mundo globalizado actual, se requieren de estrategias innovadoras que permitan intencionar de manera más efectiva el aprendizaje de los educandos despertando su interés y motivación hacia el aprendizaje con sentido y reflexión. 
En este proceso de aprendizaje deben establecerse pautas que permitan el desarrollo integral del alumno. Dentro de estas pautas, es importante mejorar y potenciar aspectos básicos como el estilo didáctico de la clase, el ambiente de la sala de clases -tanto físico como humano-, plan de estudios, estrategias evaluativas, la configuración horaria de las clases, el material didáctico entregado -digital, tradicional-. Además, mantener la preocupación por el nivel de actividad física, los hábitos de alimentación, el sueño, las emociones de los aprendices como parte del proceso natural para aprender.

Por las razones antes mencionadas, se concluye que una educación efectiva enfrenta el desafío de configurar un modelo de educación integral, centrado en el estudiante y basado en el uso de metodologías activas de enseñanza-aprendizaje (Arruti, 2015). Ello implica, reconocer la relevancia de incorporar estrategias y herramientas orientadas hacia un aprendizaje experiencial, vivencial y significativo, en desmedro de la utilización de métodos convencionales pasivos de enseñanza, que no solo arriesgan la autonomía del educando, sino que pueden llegar a inhibir el desarrollo de actitudes y competencias emprendedoras, esenciales para su formación como agente de cambio social (Gibb, 2002; Kirby, 2004; Osorio \& Pereira, 2011). Por lo demás, implica atender un rol docente renovado, capaz de generar espacios para la reflexión, la crítica, el trabajo en equipo y la autoformación, bajo un contexto donde las ideas se conviertan en acción (Sánchez, Ward, Hernández \& Florez, 2017).

\section{Referencias}

Arboccó de los Heros, M. (2016). Neurociencias , educación y salud mental. Propósitos y Representaciones, 4(1), 327-362. doi: https://doi.org/10.20511/pyr2016.v4n1.92

Arruti, A. (2015). El desarrollo del perfil del "teacherpreneur" o profesor-emprendedor en el currículum del grado de Educación Primaria: ¿Un concepto de moda o una realidad? Contextos Educativos. Revista de Educación, 19(2016), 177-194. doi: https://doi.org/10.18172/con.2770

Barrios, H. (2016). Neurociencias, educación y entorno sociocultural. Educación y Educadores, 19(3), 395-415. doi: https://doi.org/10.5294/edu.2016.19.3.5

Blakemore, S., \& Frith, U. (2007). Cómo aprende el cerebro. Las claves para la educación (2º Ed.). Barcelona: Editorial Ariel.

Blanco, J., Miguel, V., García-Castellón, C. \& Martín, P. (2017). Neurociencia y Neuropsicología Educativa. Madrid, España: Ministerio de Educación, Cultura y Deporte.

Borck, C. (2016). How we may think. Imaging and writing technologies across the history of the neurosciences. Studies in History and Philosophy of Science Part C:Studies in History and Philosophy of Biological and Biomedical Sciences, 57, 112-120. doi: https://doi.org/10.1016/j.shpsc.2016.02.006

Braidot, N. (2013). Neuromanagement y neuroliderazgo. Cómo se aplican los avances de las neurociencias a la conducción y gestión de organizaciones. Ciencias Administrativas Revista Digital, 2. doi: https://doi.org/2314-3738

Brígido, M., Caballero, A., Bermejo, M., Luisa, M. \& Mellado, V. (2009). Las emociones en la enseñanza y aprendizaje de las ciencias de maestros de primaria en formación inicial durante sus prácticas de enseñanza. International Journal of Developmental and Educational Psychology, 1(1), 399-406. Recuperado de http://www.redalyc.org/articulo.oa?id=349832320043

Carillo-Mora, P., Ramírez-Peris, J. \& Magaña-Vásquez, K. (2013). Neurobiología del sueño y su importancia: Antología para el estudiante universitario. Electrophoresis, 56(4), 5-15. Recuperado de http://www.scielo.org.mx/pdf/facmed/v56n4/v56n4a2.pdf

Comins-Mingol, I. \& París-Albert, S. (2013). Un análisis desde la filosofía para la paz. Convergencia, Revista de Ciencias Sociales, 62, 107-133.

Dávila, M., Borrachero, A., Brígido, M. \& Costillo, E. (2014). Las emociones y sus causas en el 
aprendizaje de la física y la química. INFAD, Revista de Psicología, 4(1), 287-294. Recuperado de http://www.redalyc.org/articulo.oa?id=349851787031\%0ACómo

Gago, L., \& Elgier, Á. (2018). Trazando puentes entre las neurociencias y la educación. Aportes, límites y caminos futuros en el campo educativo. Psicogente, 21(40), 476-494. doi: https://doi.org/10.17081/psico.21.40.3087

Garcés-Viera, M. V., \& Suarez-Escudero, J. C. (2014). Neuroplasticidad: aspectos bioquímicos y neurofisiológicos. CES Medicine, 28(1), 119-132.

Gibb, A. (2002). In pursuit of a new "Enterprise" and "Entrepreneurship" paradigm for learning: Creative destruction, new values, new ways of doing things and new combinations of knowledge. International Journal of Management Reviews, 4(3), 233-269. Recuperado de

http://citeseerx.ist.psu.edu/viewdoc/download?doi=10.1.1.465.9414\&rep=rep1\&type=pdf

González, D. (2006). La motivación: Varilla mágica de la enseñanza y la educación. Kaleidoscopio, 3(6), 89-94. Recuperado de http://kaleidoscopio.uneg.edu.ve/numeros/k06/k06_art01.pdf

Goswami, U. (2015). Neurociencia y Educación: ¿Podemos ir de la investigación básica a su aplicación? Un posible marco de referencia desde la investigación en dislexia. Psicología Educativa, 21, 97-105. doi: https://doi.org/10.1016/j.pse.2015.08.002

Hernández, G., \& Portolés, A. (2016). Recomendaciones de actividad física y su relación con el rendimiento académico en adolescentes de la Región de Murcia. RETOS, Nuevas Tendencias En Educación Física, Deporte y Recreación, (29), 100-104. Recuperado de http://www.redalyc.org/articulo.oa?id=345743464020

Justel, N., \& Diaz, V. (2012). Plasticidad cerebral: participación del entrenamiento musical. Suma Psicológica, 19(2), 97-108. doi: http://dx.doi.org/10.14349/sumapsi2012.1234

Kirby, D. A. (2004). Entrepreneurship education: Can business schools meet the challenge? Education + Training, 46(8/9), 510-519. doi: https://doi.org/10.1108/00400910410569632

Leopoldo, K., \& Joselevitch, C. (2018). Computational neuroscience in the study of cognitive processes. Psicologia USP, 29(1) 40-49. doi: https://doi.org/10.1590/0103656420160172

Martín-Loeches, M. (2015). Neurociencia y educación: Ya hemos alcanzado el punto crítico. Psicologia Educativa, 21(2), 67-70. doi: https://doi.org/10.1016/j.pse.2015.09.001

Meltzer, L. (2018). Executive Function in Education (Sd. Ed.). New York: The Guilford Press.

Mora, F. (2017). Neuroeducación. Madrid: Alianza Editorial.

Osorio, F. F. \& Pereira, F. (2011). Hacia un modelo de educación para el emprendimiento: Una mirada desde la teoría social cognitiva. Cuadernos de Administración, 24(43), 13-33.

Pacheco, P., Villagran, S. \& Guzmán, C. (2015). Estudio del campo emocional en el aula y simulación de su evolución durante un proceso de enseñanza-aprendizaje para cursos de ciencias. Estudios Pedagógicos (Valdivia), 199-217. doi: http://dx.doi.org/10.4067/S0718-07052015000100012

Pineda Alhucema, W. F. (2016). La Teoría de la Mente desde el Marco de la Neurociencia Cognitiva Social. Psicogente, 19(35), 8-13. Recuperado de http://revistas.unisimon.edu.co/index.php/psicogente/article/view/1381

Redolar, D. (2013). Neurociencia Cognitiva ( $1^{\circ}$ Ed.). Madrid: Panamericana.

Romero, Y. M., Monzan, F. M. \& Hernández, Y. (2014). Comunicación efectiva y desempeño laboral en Educación Básica. Negotium, 9(27), 22-33. Recuperado de http://www.redalyc.org/articulo.oa?id=78230409001

Sánchez, J., Ward, A., Hernández, B. \& Florez, J. (2017). Educación emprendedora : Estado del arte. Propósitos y Representaciones, 5(2), 401-473. doi: https://doi.org/10.20511/pyr2017.v5n2.190

Sibley, B., \& Etnier, J. (2003). The Relationship Between Physical Activity and Cognition in Children: A Meta-Analysis. Pediatric Exercise Science, 15(3), 243-256. doi: https://doi.org/10.1123/pes.15.3.243 
Valerio, G., Jaramillo, J., Caraza, R. \& Rodríguez, R. (2016). Principios de neurociencia aplicados en la educación universitaria. Formacion Universitaria, 9(4), 75-82. doi: https://doi.org/10.4067/S0718-50062016000400009 